\title{
Simvastatin Improves Outcomes of Endotoxin-induced Coagulopathy by Regulating Intestinal Microenvironment ${ }^{*}$
}

\author{
Min XU1 ${ }^{1}$, Li-li LUO ${ }^{1}$, Meng-yi DU ${ }^{1}$, Lu TANG ${ }^{1}$, Jie ZHOU ${ }^{1}$, Yu HU ${ }^{1,2,3}$, Heng MEI ${ }^{1,2,3 \#}$ \\ ${ }^{1}$ Institute of Hematology, Union Hospital, Tongji Medical College, Huazhong University of Science and Technology, Wuhan \\ 430022, China \\ ${ }^{2}$ Hubei Clinical and Research Center of Thrombosis and Hemostasis, Wuhan 430022, China \\ ${ }^{3}$ Collaborative Innovation Center of Hematology, Huazhong University of Science and Technology, Wuhan 430022, China
}

(C) The Author(s) 2022

\begin{abstract}
[Abstract] Objective: The systemic inflammatory response is regarded as the major cause of endotoxin-induced coagulopathy, which is a strong predictor of mortality in patients with severe sepsis. Simvastatin plays an important role in reducing inflammation. In addition, the gut has long been hypothesized to be the "motor" of critical illness, driving or aggravating sepsis by the increased intestinal permeability and bacterial translocation. Whether simvastatin plays a role in severe endotoxin-induced coagulopathy through the gut is unclear. Methods: In this study, mice were administered $20 \mathrm{mg} / \mathrm{kg}$ simvastatin by gavage for 2 weeks and then intraperitoneally injected with $50 \mathrm{mg} / \mathrm{kg}$ endotoxin. Twelve h later, cytokine release, coagulation dysfunction, organ damage, and survival were assessed. Besides, the intestinal barrier, permeability, bacteria abundance, and translocation were evaluated. Results: We found that the severity of endotoxin-induced coagulopathy was significantly improved in simvastatin-pretreated mice, who showed attenuated depletion of coagulation factors and platelets, decreased plasminogen activator inhibitor-1 (PAI-1) expression, reduced organ fibrin deposition, and improved survival time. Also, simvastatin reduced epithelial apoptosis and improved intestinal barrier function by upregulating antimicrobial peptides, lysozyme, and mucins. Simvastatin increased Lactobacillales counts, while the lipopolysaccharide group showed increased Desulfovibrio and Mucispirillum, which can produce harmful toxins. Finally, the decreased intestinal permeability in the simvastatin group caused reduced bacterial translocation in the organs and blood, both in terms of quantity and species. Conclusion: Simvastatin improves the prognosis of severe endotoxemia, and the intestinal microenvironment participates in this process.
\end{abstract}

Key words: endotoxemia; endotoxin-induced coagulopathy; simvastatin; inflammatory cytokines; intestinal permeability; intestinal microorganism

Severe endotoxemia occurs frequently in intensive care unit (ICU) patients and is associated with serious complications like disseminated intravascular coagulation (DIC) and even multiple organ dysfunction syndrome $(\mathrm{MODS})^{[1,2]}$. A recent study has reported that the overall mortality rate of severe sepsis is $21.5 \%$ and that the incidence of DIC in severe sepsis

Min XU, E-mail: xu_min1015@163.com

\#Corresponding author, E-mail: hmei@hust.edu.cn

${ }^{*}$ This study was supported by grants from the National Natural Science Foundation of China (No. 81873434) and the Natural Science Foundation of Hubei Province (No. 2020CFA065).

Electronic supplementary material The online version of this article (https://doi.org/10.1007/s11596022-2526-3) contains supplementary material, which is available to authorized users. is $50.9 \%{ }^{[3]}$. The gradual progression of inflammation from systemic inflammatory response syndrome (SIRS) to severe sepsis parallels the increase in the prevalence of DIC and MODS ${ }^{[4]}$. The gut has long been hypothesized to be "the motor" of critical illness, and recent studies have revealed that changes in intestinal function are crucial for the development of sepsis and that increased intestinal permeability triggers a cascade of events resulting in increased bacterial growth and risk of sepsis ${ }^{[5]}$.

The intestine is mainly composed of epithelial cells connected via tight junctions (TJs), immune cells, and intestinal microorganisms, which work together to maintain intestinal homeostasis ${ }^{[6]}$. Endotoxin-induced sepsis is related to gastrointestinal tract dysfunction and structural disorders. The most common complications are enhanced mucosal layer permeability, disturbed mucosal perfusion, development of tissue edema, 
coagulation-associated local dysregulation, bacterial translocation, and a shift in the gut microbiome ${ }^{[6]}$. For example, patients with severe SIRS had significantly lower levels of Bifidobacterium and Lactobacillus and higher levels of pathogenic bacteria than healthy volunteers ${ }^{[7]}$. A disturbance in the intestinal flora and increased intestinal permeability can promote the entrance of harmful bacteria and bacterial products into local and distant spaces that are difficult to reach under normal circumstances; potential outcomes include secondary infections, aggravated coagulopathy, and multiple organ dysfunction ${ }^{[5,8]}$. In agreement with these findings, a correlation between gut barrier dysfunction and secondary lung injury has been reported ${ }^{[9]}$. Therefore, improving intestinal microenvironment disorders is of great significance to reduce sepsis and sepsis-induced complications.

Statins, which are 3-hydroxy-3-methylglutaryl coenzyme A reductase inhibitors, reduce blood lipid levels and have pleiotropic effects, including improved endothelial dysfunction, reduced inflammation, and inhibition of platelet activation ${ }^{[10,11]}$. Although clinical and animal studies have demonstrated that statins have beneficial effects in terms of both morbidity and mortality in mild or moderate sepsis ${ }^{[12,13]}$, little is known about the changes in the intestinal tract in severe endotoxin-induced coagulopathy and whether simvastatin (SIM) can improve it by regulating intestinal microenvironment disorders. So, this study aimed to evaluate the effects and mechanism of statins on the intestinal tract in an endotoxin mouse model.

\section{MATERIALS AND METHODS}

\subsection{Animals}

Male C57BL/6J mice aged 8 weeks were purchased from Beijing HFK Bio-Technology Co., Ltd. (China) and housed at the specific pathogen-free facility of Huazhong University of Science and Technology. All animal care and operations were performed following national and European guidelines for the handling and use of experimental animals. Animal experiments were approved by the Animal Experimentation Ethics Committee of Huazhong University of Science and Technology (reference number: 2266).

\subsection{Establishment of Animal Model}

After a one-week adaptation period, the mice were randomly divided into three groups: control, lipopolysaccharide (LPS), and LPS+SIM groups. Mice in the LPS+SIM group were given SIM (MedChem Express, USA) daily by gavage for 2 weeks, and the others were administered sterile water. Two weeks later, mice in the LPS and LPS+SIM groups were intraperitoneally (i.p.) injected with LPS (Escherichia coli, O55: B5, Sigma, USA), and the control mice were injected with normal saline. We choose $20 \mathrm{mg} /$ $\mathrm{kg} \mathrm{SIM} \mathrm{as} \mathrm{the} \mathrm{treatment} \mathrm{dose,} \mathrm{which} \mathrm{has} \mathrm{an} \mathrm{effect} \mathrm{on}$ microbiota modulation, and $50 \mathrm{mg} / \mathrm{kg}$ LPS to induce coagulopathy, which has been used to establish the DIC model in previous studies ${ }^{[2,14,15]}$. Food and water were provided ad libitum, and volume support $(0.9 \%$ $\mathrm{NaCl}, 0.3 \mathrm{~mL}$ ) was applied subcutaneously after the induction of LPS and every $4 \mathrm{~h}$ thereafter. The animals in each group were sacrificed at $12 \mathrm{~h}$ after the LPS injection, and a few mice died at $6 \mathrm{~h}$. For the survival analysis, mice were monitored every $4 \mathrm{~h}$ for 7 days. Blood was drawn from the inferior cava of anesthetized mice ( $1 \%$ pentobarbital sodium, $50 \mathrm{mg} / \mathrm{kg}$, i.p.) and anticoagulated with ethylenediaminetetraacetic acid $(10 \mathrm{mmol} / \mathrm{L})$ or citrate (final concentration, $3.2 \%)$. Samples were centrifuged at $3000 \times \mathrm{g}$ for $20 \mathrm{~min}$ at $4^{\circ} \mathrm{C}$, and then the supernatant was frozen at $-80^{\circ} \mathrm{C}$ until analysis. Tissues were fixed or stored at $-80^{\circ} \mathrm{C}$ for histological examination or RNA extraction. Fresh stool samples were collected from the cecum, snapfrozen, and stored at $-80^{\circ} \mathrm{C}$ for subsequent analysis.

\subsection{Blood Assays}

Coagulation-related markers in plasma, including prothrombin time (PT), activated partial thromboplastin time (APTT), fibrinogen, fibrin/fibrinogen degradation product (FDP) levels, and antithrombin III (ATIII) activity, were measured on an automated STA-R Evolution Analyzer (Stago, France). The platelet count (PLT) was determined on an automated animal blood counter (PE-6800VET, PROKAN Co., Ltd., China). Serum levels of blood urea nitrogen (BUN), creatinine (CR), aspartate aminotransferase (AST), and alanine aminotransferase (ALT) were determined with an Automated Chemistry Analyzer (Chemray-240, Rayto, China). Plasma concentrations of cytokines including interleukin (IL)-6, IL-10, monocyte chemoattractant protein-1 (MCP-1), interferon- $\gamma$ (IFN- $\gamma$ ), and tumor necrosis factor- $\alpha$ (TNF- $\alpha$ ) were determined with a Mouse Inflammation Cytometric Bead Array kit (No. 552364, BD Pharmingen, USA) according to the manufacturer's instructions. Plasma levels of mouse IL- $1 \beta$ and IL-18 were measured using ELISA kits obtained from BD Pharmingen (No. 560232; No. 216165, USA). We also measured various hemostatic endothelial molecular markers, including soluble thrombomodulin (TM), thrombin-antithrombin complex (TAT), and plasminogen activator inhibitor-1 (PAI-1), using appropriate ELISA kits (Abcam, UK).

\subsection{Histological Examination}

Ileum tissue samples were immediately washed with cold $0.9 \%$ saline solution and fixed in $4 \%$ paraformaldehyde for at least $24 \mathrm{~h}$. The fixed samples were embedded in paraffin and sectioned. The sections were deparaffinized, rehydrated, and stained with hematoxylin and eosin for histological assessment of the intestinal mucosa. Changes in the intestinal mucosa were observed with an optical microscope (Leica, 
Germany).

\subsection{Immunohistochemistry}

Tissue samples were fixed in $4 \%$ paraformaldehyde and embedded in paraffin. Then, the sections were immunostained with antibodies against fibrinogen (1:20 000 dilution, Abcam, UK), TF (1:400 dilution, Abcam, UK), and Ly-6G (1:200 dilution, BD Pharmingen, USA), according to the manufacturers' protocols.

1.6 Quantitative Real-time Polymerase Chain Reaction

TRIzol reagent (Takara, China) was used to isolate total RNA from tissues and cells, according to the manufacturer's instructions. cDNA was generated using a PrimeScript RT reagent kit (Takara, China), and Quantitative real-time polymerase chain reaction (qRT-PCR) was performed using a SYBR Green RTPCR kit (Takara, Japan). GAPDH was used as an internal control, and the primers are shown in table S1. All PCRs were run on an ABI 7500 FAST Real-Time PCR System (Applied Biosystems, USA), and each sample was tested in triplicate. Relative expression was calculated using the comparative $2^{-\Delta \Delta \mathrm{CT}}$ method.

\subsection{Western Blot Analysis}

Frozen ileum segments were homogenized, and the supernatant was collected. The total protein concentration was measured via a BCA protein assay. A total of 20-30 $\mu \mathrm{g}$ of ileum tissue lysate was separated on $10 \%$ SDS-polyacrylamide gels and transferred to polyvinylidene fluoride membranes. After being blocked, the membranes were incubated overnight at $4{ }^{\circ} \mathrm{C}$ with primary antibodies against the following proteins: $\mathrm{Bax}$ (1:6000), Bcl-2 (1:1000), and caspase 3 (1:1000) (Proteintech Group, USA). The membranes were washed three times and incubated with horseradish peroxidase-conjugated goat antirabbit secondary antibody (1:1000, Proteintech Group, USA) for $60 \mathrm{~min}$ at room temperature. Finally, the pixel density was detected with a chemiluminescence system (Pierce, USA).

\subsection{In Vivo Intestinal Permeability Assay}

FITC-D4000 (FD-4, Sigma-Aldrich, USA) dissolved in saline to $50 \mathrm{mg} / \mathrm{mL}$ was given to the mice via gavage at a dose of $600 \mathrm{mg} / \mathrm{kg}$. At $1 \mathrm{~h}$ after gavage, blood was collected from anesthetized mice and centrifuged at $12000 \mathrm{r} / \mathrm{min}$ at $4^{\circ} \mathrm{C}$ for 10 $\min ^{[16]}$. A total of $50 \mu \mathrm{L}$ of plasma was diluted 1:2 with phosphate-buffered saline (PBS, pH 7.4). The concentration of FD-4 was measured using a MultiDetection Microplate Reader (Synergy HT, Bio-Tek software KC4, USA) at an excitation wavelength of $493 \mathrm{~nm}$ and an emission wavelength of $518.5 \mathrm{~nm}$. Standards (range: $1000-7.8125 \mathrm{ng} / \mathrm{mL}$ ) were obtained by diluting the FD-4 gavage stock solution with plasma from control animals.

\subsection{Bacteriological Analysis}

Bacterial translocation was determined by culturing homogenized mesenteric lymph nodes
(MLNs), lungs, liver, spleen, kidney, blood, and peritoneal fluid (PLF). PLF was collected after rinsing the abdomen with $1 \mathrm{~mL}$ of isotonic saline, allowing the saline to remain within the peritoneal cavity for at least $30 \mathrm{~s}$. Tissue samples were aseptically removed at $12 \mathrm{~h}$ after LPS administration, weighed, and homogenized in PBS to achieve a $50 \mathrm{mg} / \mathrm{mL}$ concentration. Next, $0.1 \mathrm{~mL}$ aliquots of the samples were inoculated onto a Columbia Nutrient Agar plate with 5\% sheep blood to grow total bacteria. Plates were cultured anaerobically in a $37^{\circ} \mathrm{C}$ incubator for $48 \mathrm{~h}$, and colonies were counted. Bacterial numbers are expressed as colony-forming units per gram of tissue or per $\mathrm{mL}$ of blood or fluid.

\subsection{S rDNA Sequencing}

Frozen feces samples and lung samples were used to determine the gut microbiome profile. Genomic DNA was isolated using a PowerSoil ${ }^{\circledR}$ DNA Isolation Kit (MO BIO Laboratories Inc., USA) according to the manufacturer's protocol. The high-throughput sequencing library was constructed and sequenced using the Illumina MiSeq platform by GENEWIZ (China). With 30-50 ng of DNA as a template, a series of PCR primers designed by Jin Weizhi were employed to amplify prokaryotic $16 \mathrm{~S}$ rDNA, including the two highly variable regions V3 and V4, using a MetaVx Library Preparation kit (GENEWIZ, China).

Sequences longer than $200 \mathrm{bp}$ were retained, and sequence clustering was performed using VSEARCH (1.9.6) at the $97 \%$ similarity level ${ }^{[17]}$. The most abundant sequence in each operational taxonomic unit was selected, and the Shannon index was calculated by random sampling of sample sequences. Based on the Bray-Curtis inter-sample distance matrix, the principal coordinate analysis (PCoA) was visualized to show the $\beta$ diversity. The abundance data for each sample were normalized and log-transformed. The differences in microbial composition among different samples were assessed by analysis of similarities (ANOSIM).

\subsection{Cell Culture}

Human epithelial colorectal adenocarcinoma (Caco-2) cells were cultured in Dulbecco's modified Eagle's medium/high glucose (Gibco, USA) supplemented with $10 \%$ fetal bovine serum, penicillin, and streptomycin. The cells were maintained in a 5\% $\mathrm{CO}_{2}$ incubator at $37^{\circ} \mathrm{C}$, and the culture medium was changed every 1-2 days. Trypsinized cells were added to 6-well tissue culture-treated plates $(2000 \mu \mathrm{L}$; Costar, Corning, USA) at $2 \times 10^{5}$ cells $/ \mathrm{cm}^{2}$. After 14 days, a confluent cell monolayer was obtained. The cells were stimulated with different concentrations of $\operatorname{SIM}(0,500$ $\mathrm{nmol} / \mathrm{L}, 1 \mu \mathrm{mol} / \mathrm{L}, 10 \mu \mathrm{mol} / \mathrm{L}$, or $20 \mu \mathrm{mol} / \mathrm{L})$ for 60 $\mathrm{h}$ and then cotreated with LPS $(20 \mu \mathrm{g} / \mathrm{mL})$ for $12 \mathrm{~h}$. Finally, the cells were collected for qPCR experiments. 1.12 Statistical Analysis

All data in this study are presented as the mean $\pm \mathrm{SEM}$, and $P<0.05$ was considered to indicate 
statistical significance in all experiments. If the data had a normal distribution and homogeneity of variance, one-way ANOVA followed by the Newman-Keuls post hoc test or Dunnett's $t$-test for multiple group comparisons was performed. Otherwise, a KruskalWallis $\mathrm{H}$ test was used. For comparisons of two groups, a $t$-test was used. Also, mortality data were reanalyzed and presented as a Kaplan-Meier survival curve. Bacteriologic culture results were analyzed with Fisher's exact test. All statistical analyses were performed using SPSS (version 19.0, USA), and the figures were generated with Prism 5 (GraphPad, USA) or Adobe Illustrator (CS5, USA) software.

\section{RESULTS}

\subsection{Simvastatin Improves Outcomes of Endotoxin- induced Coagulopathy}

SIM improves endotoxin-induced coagulopathy, reduces organ failure, and extends survival. Prophylactic administration of SIM helped shorten the prolongation of PT and APTT. The PLT, fibrinogen, and AT III levels dropped sharply at $12 \mathrm{~h}$ after the injection of endotoxin, and these decreases were significantly alleviated in the SIM group. After endotoxin injection, the PLT concentration was $(205 \pm 28) \times 10^{9} / \mathrm{L}$ in the LPS group and $(305 \pm 44) \times 10^{9} / \mathrm{L}$ in the LPS + SIM group, the corresponding fibrinogen levels were $1.6 \pm 0.2$ $\mathrm{g} / \mathrm{L}$ and $2.5 \pm 0.3 \mathrm{~g} / \mathrm{L}(P<0.01)$, and the AT III levels were $44 \% \pm 2.5 \%$ and $54 \% \pm 1.6 \%$, respectively. PAI-1 increased significantly after endotoxin administration and decreased in the SIM group. TAT and TM had no significant differences between the two groups (fig. 1A). Histologic studies showed elevated TF levels in lung tissue in the LPS group as compared with the LPS+SIM group (fig. 1B). Additionally, more extensive fibrin deposition was observed in various organs at $12 \mathrm{~h}$ after endotoxin administration, while it was significantly inhibited by SIM pretreatment (fig. 1C). Mice treated with SIM had significantly reduced serum ALT $(P<0.05)$, AST $(P<0.01)$, and CR $(P<0.01)$ levels as compared with the LPS group (fig. 1D). However, there was no significant difference in BUN between the two groups.

No deaths occurred in the control group. The survival curves for mice in the LPS group sharply declined, and all mice died within $20 \mathrm{~h}$. However, in the SIM-treated group, the survival rate increased by $20 \%$ at $48 \mathrm{~h}$ and $13.3 \%$ at $72 \mathrm{~h}(P<0.001$, fig. 2$)$. Continued observations revealed that the two surviving mice gradually recovered their appetite and activity from day 6.

SIM alters endotoxin-induced inflammatory responses. Plasma proinflammatory and antiinflammatory factor levels, including IL-1 $\beta$, IL-6, TNF- $\alpha$, MCP-1, IFN- $\gamma$, IL-18, and IL-10, increased significantly in the LPS group. SIM pretreatment decreased the plasma proinflammatory cytokine levels and increased IL-10 levels as compared with LPS alone (fig. 3A). This phenomenon was verified in lung tissue at the mRNA level (fig. 3B). The LPS group had a large number of infiltrating granulocytes in the lungs, which was significantly improved in the SIMpretreated group (fig. 3D).

The mRNA levels of inflammatory cytokines in the intestine were also detected. Unexpectedly, SIM administration did not decrease the LPS-induced increases in IL-1 $\beta$, MCP-1, and IL-6 mRNA levels. The TNF- $\alpha$ mRNA levels were significantly upregulated and the anti-inflammatory cytokine IL-10 was downregulated by SIM administration as compared with LPS alone (fig. 3C). This finding was inconsistent with the regulatory effect of SIM in the lung tissue.

\subsection{Simvastatin Improves Intestinal Microenviron- ment Disorders}

SIM improves intestinal physical barrier functions. The intestinal mucosa of mice treated with saline was intact, with well-ordered villi. However, in the LPS group, the intestinal villi were disrupted, atrophic, or even ruptured at locations of necrotic epithelial cells, and the mucosa was edematous. The damage was significantly alleviated in the SIM-pretreated group; although the small intestinal villi were swollen and deformed, there was no fracture in the barrier of the villi (fig. 4A). Decreased gut epithelial apoptosis is associated with an improved survival rate in patients with sepsis ${ }^{[18]}$. In our study, SIM reversed the LPSinduced upregulation of caspase 3 and increased the expression of Bcl-2 at the protein level, the Bax/Bcl-2 ratio was increased in the LPS group and decreased in the LPS+SIM group, and these results were verified at the mRNA level (fig. 4B and 4C).

TJs are a large family of proteins that constitute the intestinal mechanical barrier. In our study, the mRNA levels of ZO-1 and JAM did not change significantly in the LPS group but were markedly increased by SIM treatment; occludin and claudin-4 levels were sharply decreased in both the LPS and LPS+SIM groups (fig. 4D). However, in Caco-2 cells, the ZO1, JAM-A, occludin, and claudin-4 levels increased in a concentration-dependent manner in the SIM-treated group (fig. 4E).

Simvastatin alters intestinal chemical barrier functions. Chemical barriers, including lysozyme, digestive enzymes, mucopolysaccharide, and antimicrobial peptides, are also important components of the intestinal mucosal barrier. As expected, Reg3b, Defb1 , and angiogenin-1 were significantly downregulated after $12 \mathrm{~h}$ of endotoxin treatment, but these effects were significantly reversed in the SIM pretreatment group. However, Reg3g increased 4-fold in both the LPS and LPS+SIM groups (fig. 4F). 

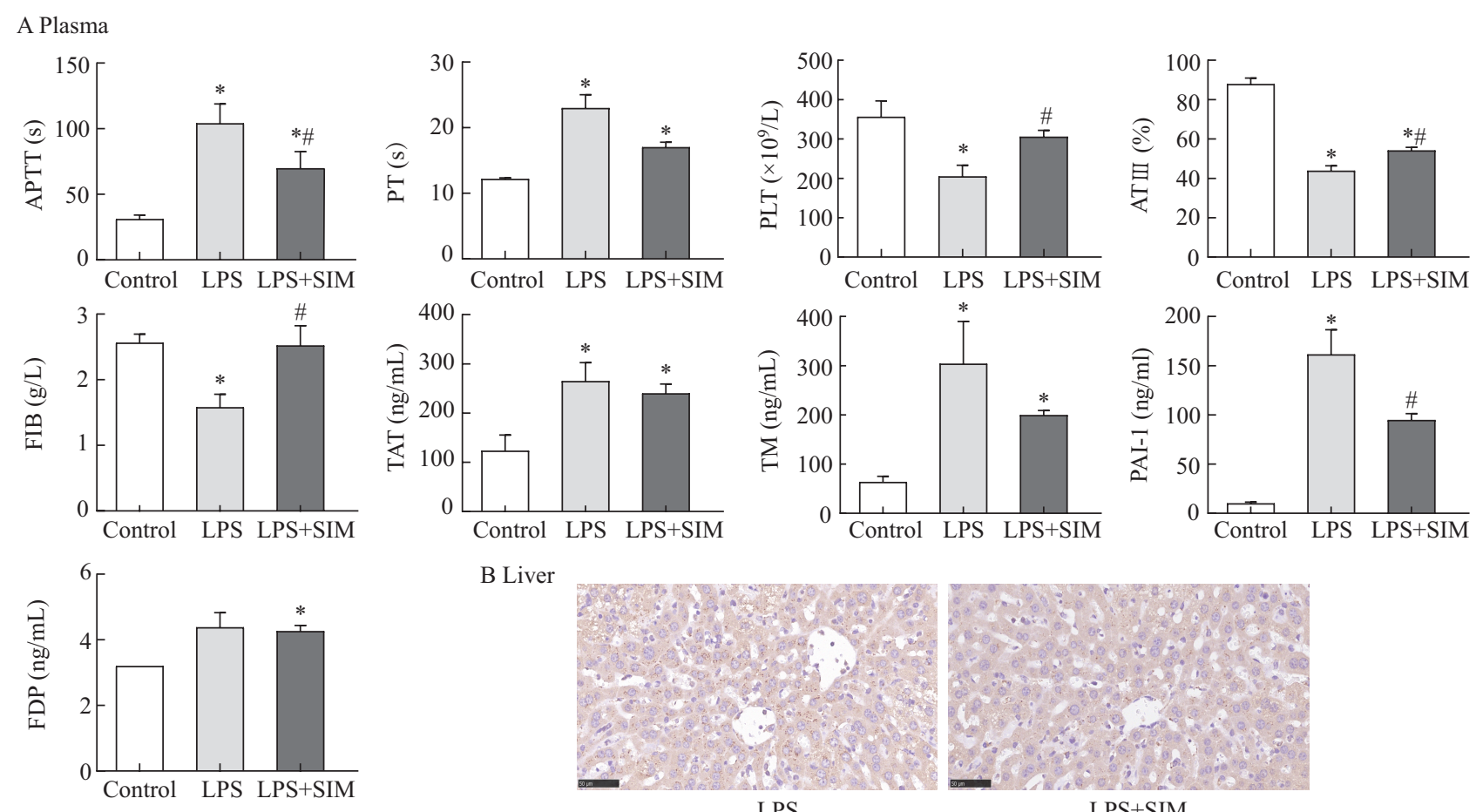

B Liver
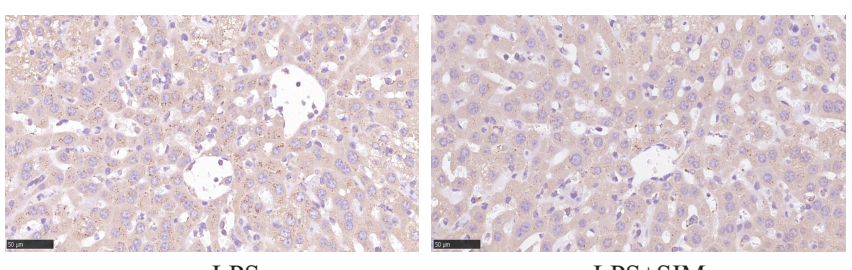

LPS

LPS+SIM

C

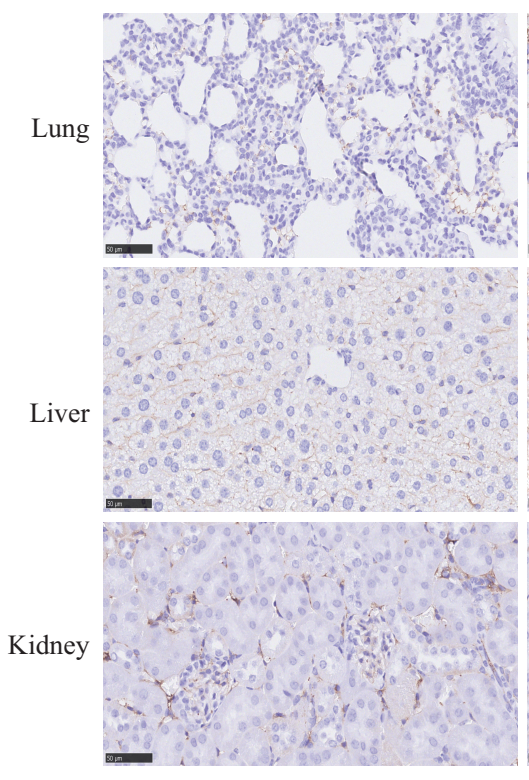

Control

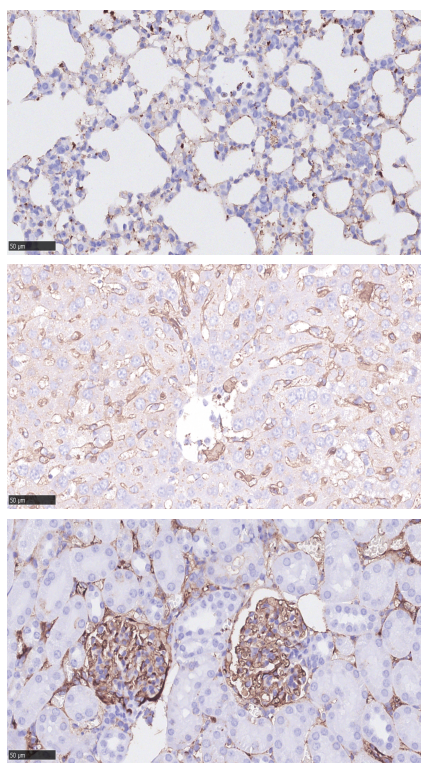

LPS

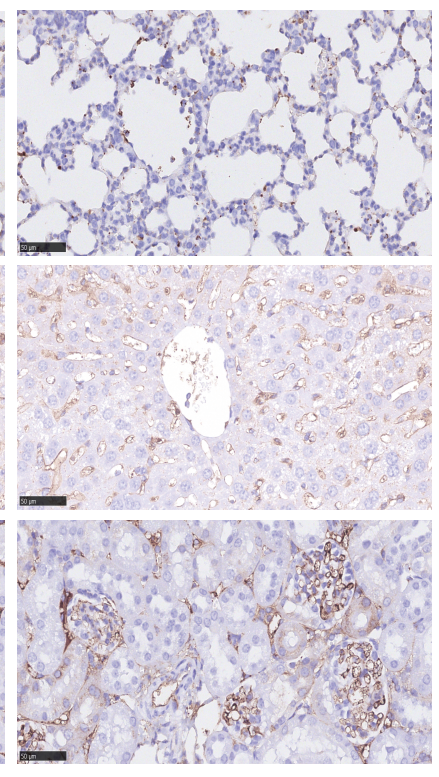

LPS+SIM
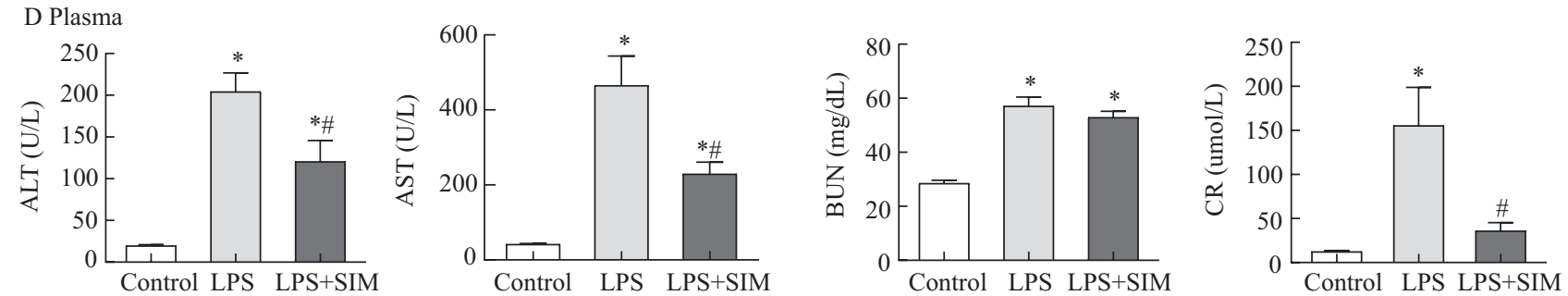

Fig. 1 Simvastatin improves LPS-induced coagulopathy and reduces organ failure at $12 \mathrm{~h}$

A: The PT, APTT, fibrinogen level, FDP level, and ATIII activity were measured with an automated blood coagulation analyzer, PLT was measured on an automated animal blood counter, and the plasma levels of TAT, PAI-1, and TM were measured using ELISA kits. B: immunohistochemical analysis of TF expression in the indicated groups. Representative images (magnification, $400 \times$ ) are shown. C: fibrin staining of the lungs, kidneys, and liver in each group. D: plasma levels of ALT, AST, CR, and BUN in the indicated groups. The data are presented as the mean $\pm \operatorname{SEM}(n=8) .{ }^{*} P<0.05$ vs. the control group, ${ }^{\#} P<0.05$ comparisons between the LPS group and the LPS+SIM group. PT: prothrombin time; APTT: activated partial thromboplastin time; FDP: fibrinogen degradation products; AT III : antithrombin III activity; PLT: platelet counts; TAT: thrombin-antithrombin complex; PAI-1: plasminogen activator inhibitor-1; TM: thrombomodulin; TF: tissue factor; AST: aspartate aminotransferase; ALT: alanine aminotransferase; BUN: blood urea nitrogen; CR: creatinine; LPS: lipopolysaccharide; SIM: simvastatin 


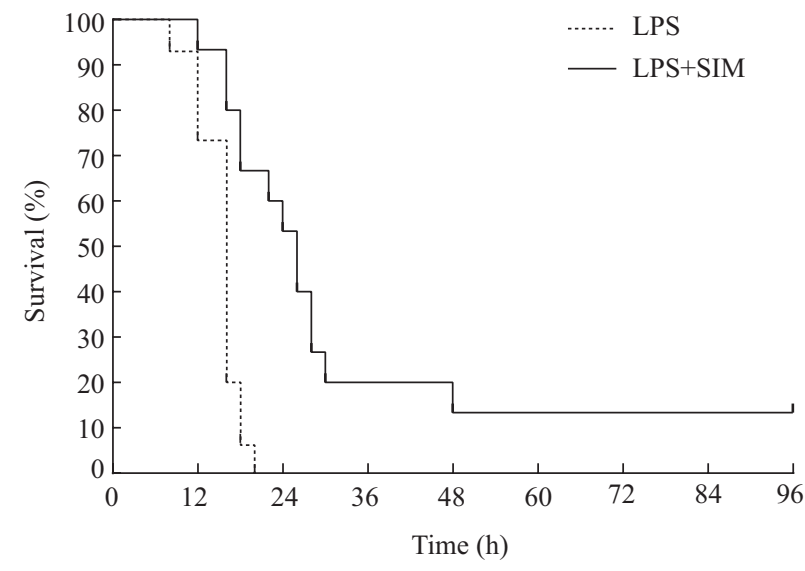

Fig. 2 Survival curves

The survival time of simvastatin-pretreated mice (broken line) was profoundly increased compared to that of untreated mice after endotoxin administration. No deaths occurred in the control group $(P<0.001, n=15)$.

The mucus is another barrier that can protect epithelial cells from digestive enzymes ${ }^{[19]}$. Core 3ß1,3-N-acetylglucosaminyltransferase (C3gnt) is responsible for glycosylation of intestinal mucins, and muc 2 can disassociate pathogenic and commensal bacteria $^{[19,20]}$. The mRNA levels of muc2 and C3gnt were significantly decreased in the LPS group but increased in the SIM group. Additionally, the expression levels of intestinal alkaline phosphatase (Iap, detoxifies bacterial LPS) were increased in the SIM treatment group. However, no significant changes in the levels of lysozyme (protecting against bacterial infection) were observed (fig. 4G).

SIM alters intestinal biological barrier. The 16sDNA sequence was used to explore the microbiota composition in the three groups. The ANOSIM of PCoA matrix scores indicated a significant separation in the microbiota composition in the three groups $(P<0.05$; fig. 5A). LPS treatment increased the community diversity, as shown by the Shannon index, and statin treatment decreased the community diversity; however, there were no significant differences among groups (fig. 5C). In both of the treated groups, the top two phyla were Firmicutes and Bacteroidetes. Compared to the control group, the LPS group showed an increased abundance of Firmicutes $(65.6 \%$ vs. $45.5 \%)$ and Proteobacteria (10.6\% vs. $2.8 \%)$ as well as a decreased abundance of Bacteroidetes (20.5\% vs. 45.7\%). In contrast, the SIM-treated group showed decreased Firmicutes (60.5\%) and Proteobacteria (3.5\%) and increased Bacteroidetes (31.7\%) compared to the LPS group and was more similar to the control group. These data suggest that the SIM-treated mice had a different gut microbiota composition from the LPS-treated mice but a composition similar to the control mice (fig. 5B).

Distinctive gut microbiota compositions in the indicated groups were also identified by linear discriminant analysis. Endotoxin treatment induced a marked increase in the abundance of harmful bacteria, including Desulfovibrio $(P<0.05)$ in the Proteobacteria phylum and Mucispirillum $(P<0.05)$ in the Deferribacteres phylum. LPS also increased the abundance of Ruminococcaceae (Firmicutes phylum). Conversely, statin therapy triggered a considerable enrichment of bacteria in the class Bacilli; however, this expansion was not class-wide but rather was due to the expansion of Lactobacillaceae (fig. 5D and 5E).

SIM decreased gut permeability and inhibited translocation of intestinal flora. Intestinal permeability was measured at $0 \mathrm{~h}, 6 \mathrm{~h}$, and $12 \mathrm{~h}$ after LPS injection. There was no difference in intestinal permeability between the LPS and LPS+SIM groups at baseline; at $6 \mathrm{~h}$, the permeability was significantly lower in the LPS+SIM group than in the LPS group, but this difference was not obvious at $12 \mathrm{~h}$ (fig. $4 \mathrm{H}$ ).

To further explore whether SIM pretreatment reduced bacterial translocation, we adopted bacterial cultures in organs and blood. We found that the positive rate of bacterial culture in the LPS group was higher than that in the LPS+SIM group, especially in the liver, kidney, and blood (fig. 6A). We further counted the number of positive bacterial colonies and found that in the liver, kidney, lung, spleen, and blood, the content of translocated bacteria in the LPS group was significantly higher than that in the LPS+SIM group (fig. 6B). However, no significant difference was found in colony units between MLNs and FLF, which are outposts for bacterial translocation.

Also, 16sDNA sequencing revealed that the lung tissue microbial amplification success rate reached $100 \%$ in the LPS group, which was higher than that in the LPS+SIM group (62.5\%) and consistent with the bacterial culture results (fig. 6C). The LPS and LPS+SIM groups showed different distributions of translocated microbes in the lung tissues, with species and number being more abundant in the LPS group (fig. 6D-6F). The significantly increased flora in the LPS group included Escherichia-Shigella, Helicobacter, Faecalibacterium, Lachnospiraceae, Bacteroides, and Bifidobacterium, while Lactococcus and Streptococcus were higher in the LPS+SIM group (fig. $6 \mathrm{G}$ and $6 \mathrm{H}$ ).

\section{DISCUSSION}

In this study, we systematically explored the effects of statins and found that LPS alone induced more serious coagulopathy and organ failure, while SIM attenuated the progression of coagulation dysfunction by enhancing anticoagulant levels and fibrinolysis, alleviated organ microthrombosis, and prolonged the survival time. In severe endotoxin-induced sepsis or coagulopathy, the gut is severely damaged. However, SIM pretreatment reduced the intestinal barrier damage 

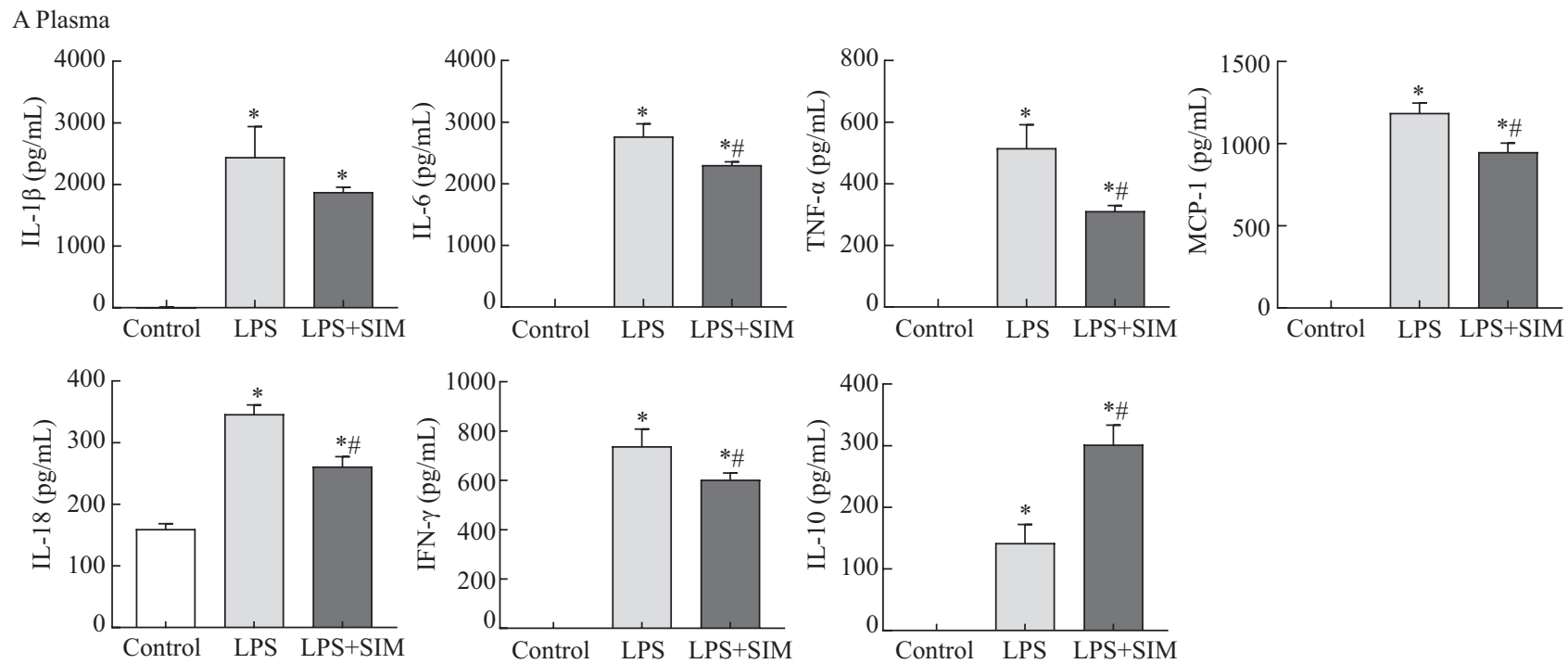

Bumes

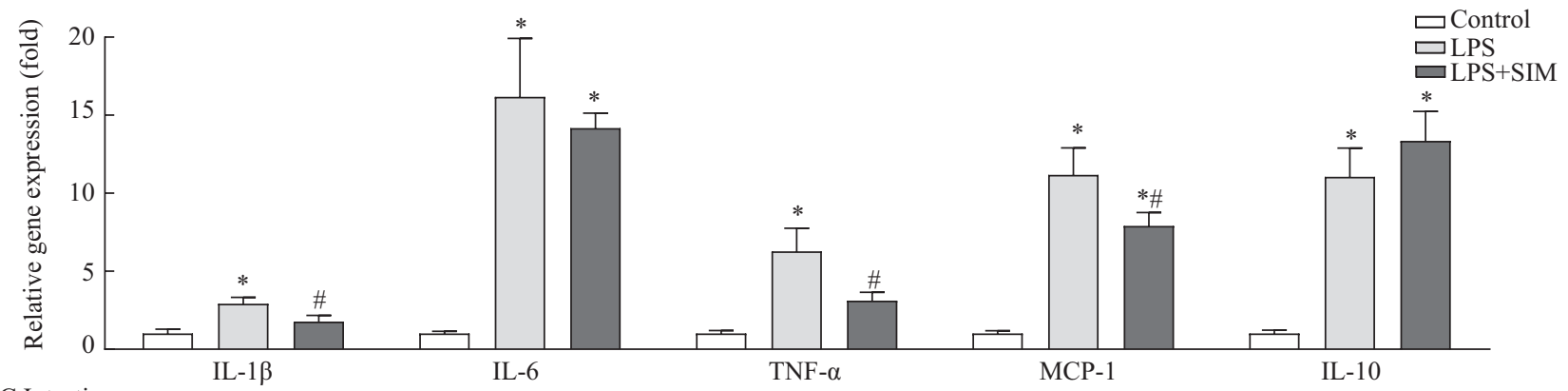

C Intestine

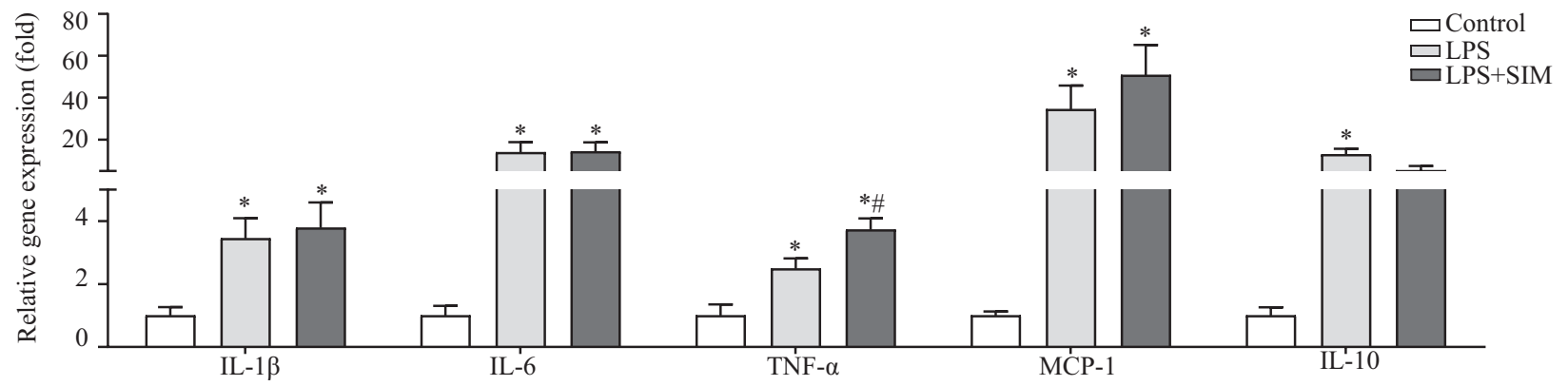

D Lung

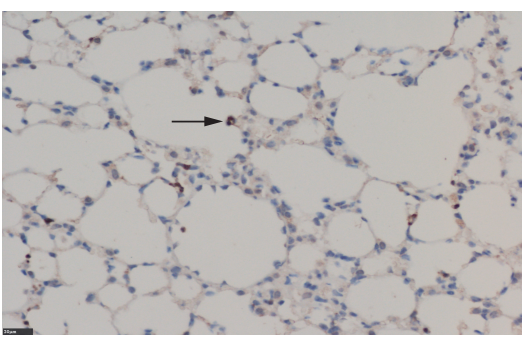

Control

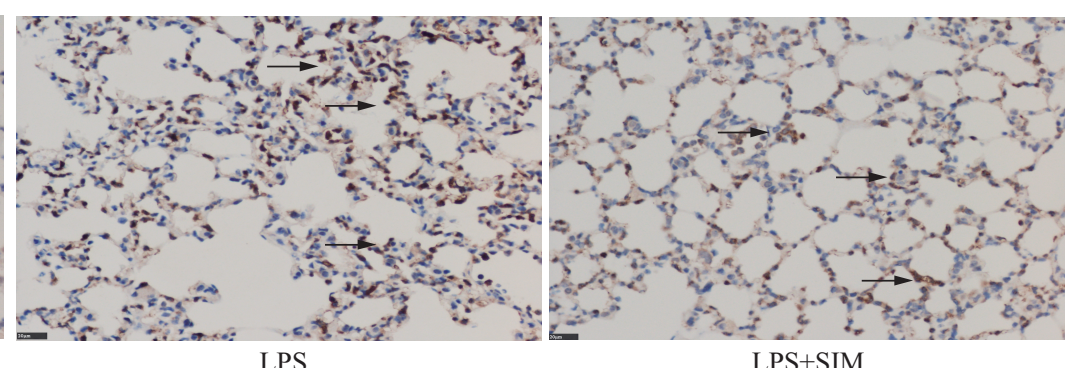

LPS+SIM

Fig. 3 Simvastatin pretreatment alters LPS-induced inflammatory responses at $12 \mathrm{~h}$

A: The plasma levels of inflammatory factors in the indicated groups were measured using ELISAs or a Mouse Inflammation Cytometric Bead Array kit. B: The mRNA levels of inflammatory factors in the lung were detected by qPCR. C: The mRNA levels of inflammatory factors in the intestine were detected by qPCR. D: anti-granulocyte staining of lung tissues. Representative images (magnification, 400×) are shown. The data are presented as the mean \pm SEM $(n=8)$. ${ }^{*} P<0.05$ vs. the control group, ${ }^{\#} P<0.05$ comparisons between the LPS group and the LPS+SIM group. ELISA: enzyme linked immunosorbent assay; LPS: lipopolysaccharide; SIM: simvastatin

and altered the intestinal microbial composition, with increased amounts of Lactobacillales and decreased amounts of Desulfovibrio and Mucispirillum. With the above changes, the intestinal permeability was reduced 


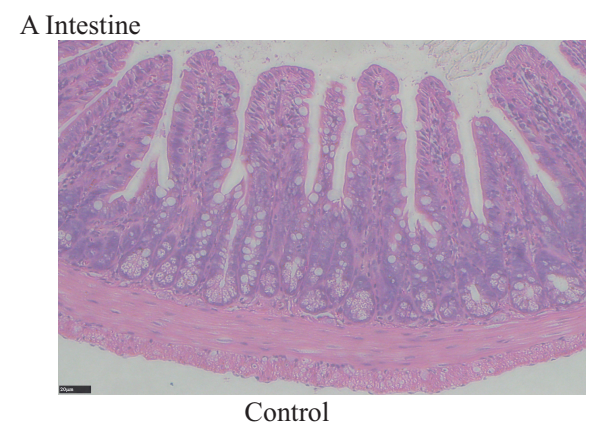

B Intestine

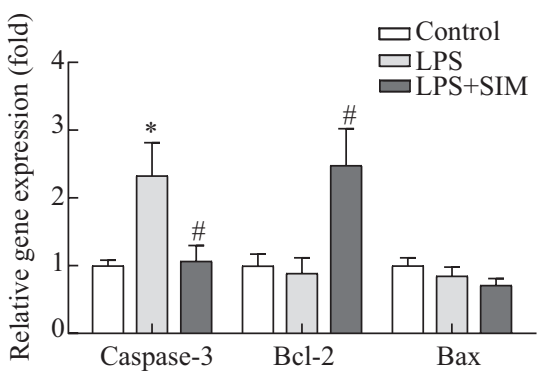

D Intestine

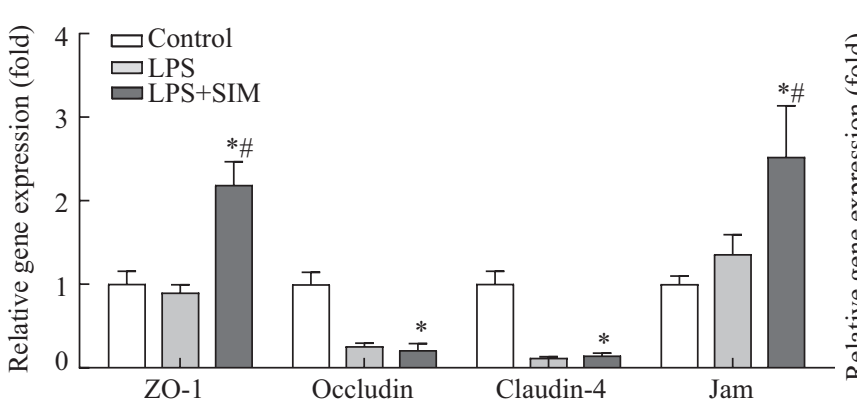

F Intestine

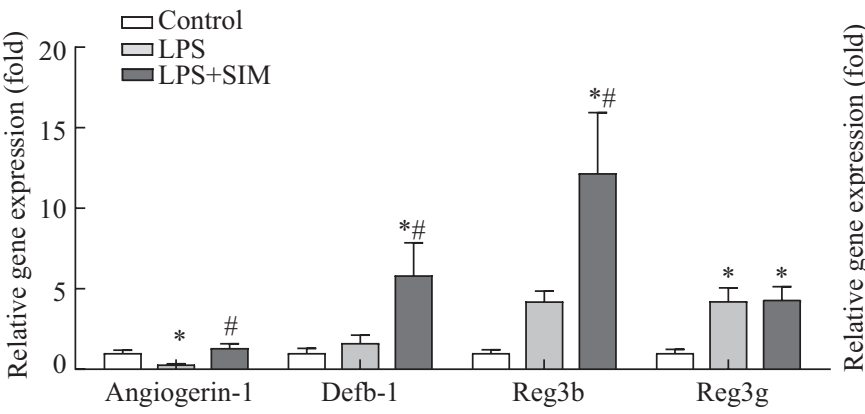

C Intestine
$\mathrm{G}$ Intestine

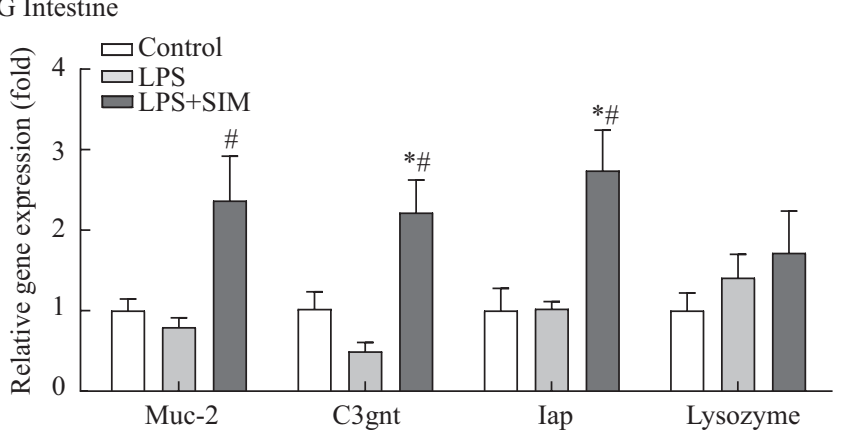

LPS
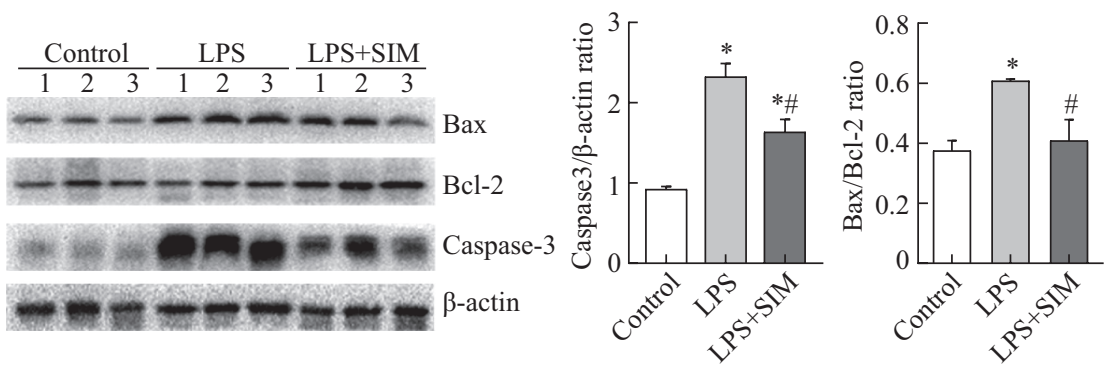

E Caco-2

שControl שPS $20 \mu \mathrm{g} / \mathrm{mL}$

口SIM $500 \mathrm{nmol} / \mathrm{L}+\mathrm{LPS} 20 \mu \mathrm{g} / \mathrm{mL}$

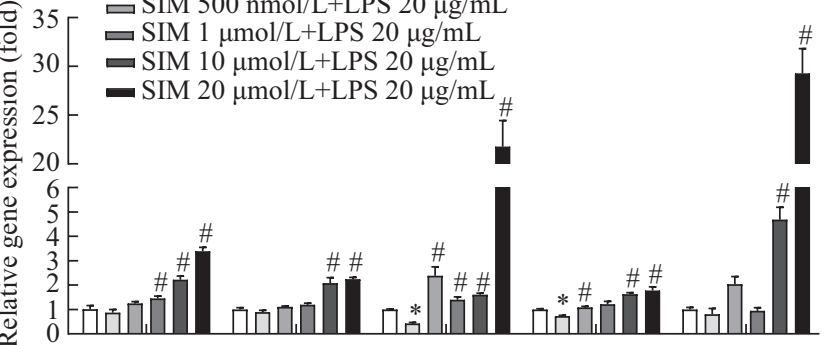

ZO-1

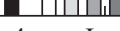

Lysozyme

H Plasma

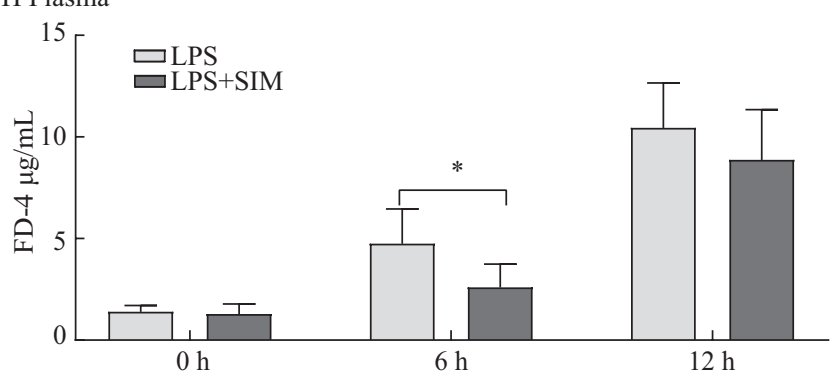

Fig. 4 Simvastatin alleviates LPS-induced intestinal barrier damage at $12 \mathrm{~h}$

A: Hematoxylin and eosin staining of ileal segments in the indicated groups (magnification, 200×). B and C: qRT-PCR (B) and Western blotting $(\mathrm{C})$ were used to determine the expression of caspase 3, Bcl-2, and Bax at the mRNA level and the protein level. The mRNA levels of TJ components (D), antibacterial peptides (F), mucin (G), and TJ components (E) in Caco-2 cells are shown. H: FITC-Dextran (FD-4) levels in plasma. The data are presented as the mean \pm SEM $(n=8) .{ }^{*} P<0.05 v s$. the control group, ${ }^{\#} P<0.05$ comparisons between the LPS group and the LPS+SIM group. qRT-PCR: quantitative real-time polymerase chain reaction; TJ: tight junction; FD-4: fluorescein 5-isothiocyanate dextran 4000; LPS: lipopolysaccharide; SIM: simvastatin 

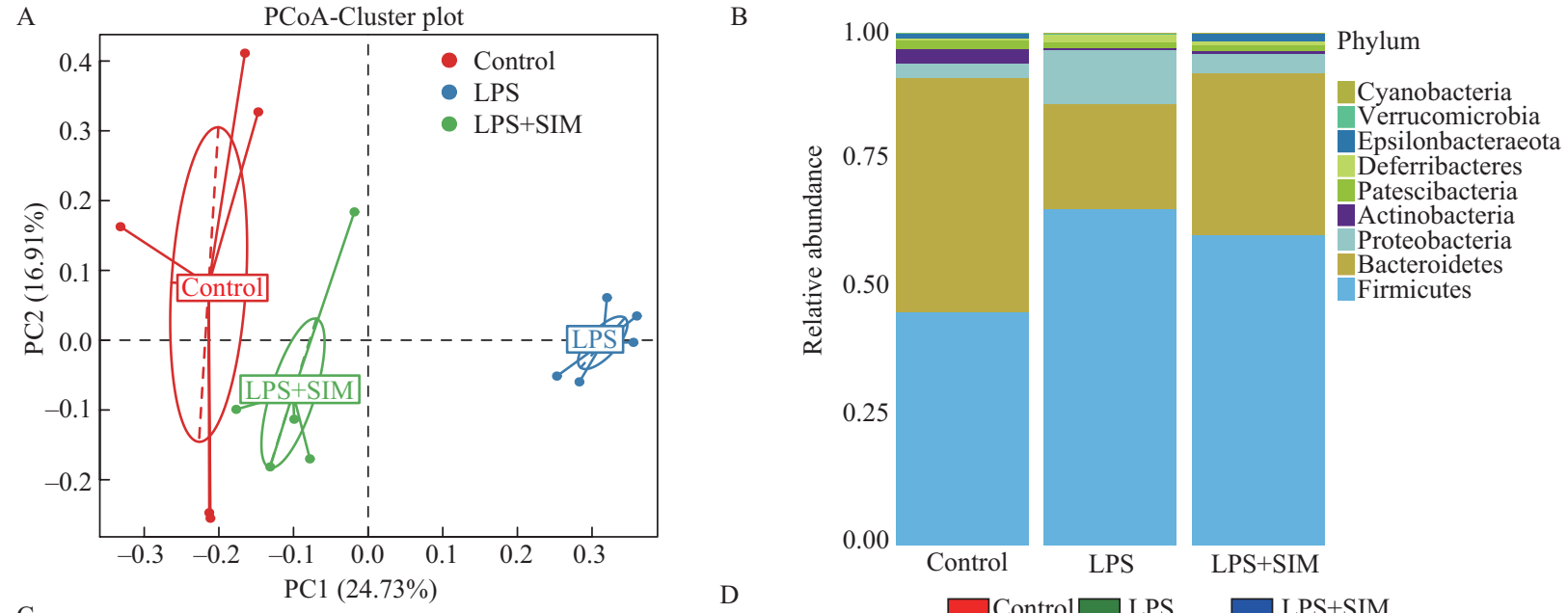

D
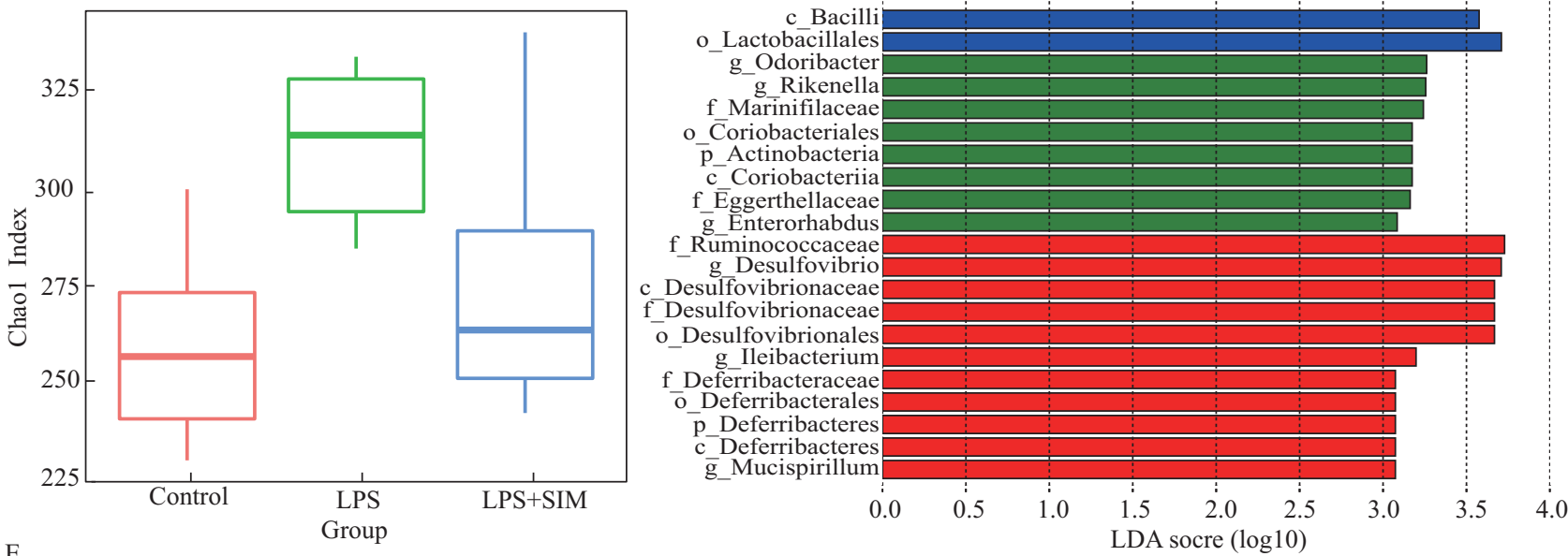

E
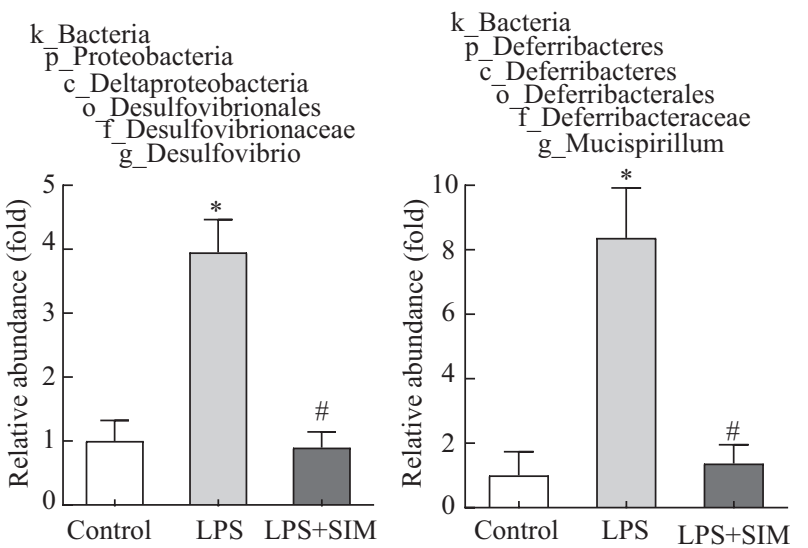
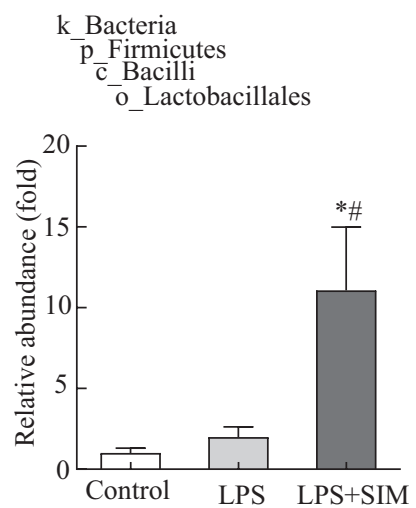
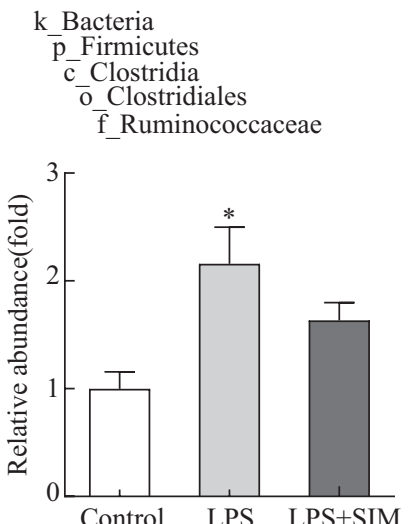

Fig. 5 Simvastatin-pretreated mice show different gut microbiota compositions.

A: PCA score plot. B: gut microbiota distribution at the phylum level. C: Chao1 index. D: distinctive gut microbiota composition revealed by LDA in the indicated groups. E: comparison of relative taxonomic abundance among the indicated groups ( $n=5)$. ${ }^{*} P<0.05$ vs. the control group, ${ }^{\#} P<0.05$ comparisons between the LPS group and the LPS+SIM group. PCA: principal coordinate analysis; LDA: linear discriminant analysis; LPS: lipopolysaccharide; SIM: simvastatin

in the simvastatin group, and the translocation of intestinal flora in organs and blood was inhibited.

SIM pretreatment reduced inflammation as well as improved the coagulation disorder and mortality rates in severe endotoxemia in this study. TF levels have been shown to increase 125-fold in blood monocytes in healthy humans after the administration of endotoxin at a low dose ${ }^{[21]}$, and statins can reduce TF expression ${ }^{[22]}$.
In our experiments, SIM pretreatment alleviated LPSinduced fibrinogen consumption; however, fibrinogen regulation by statins is controversial, and discrepancies may be related to different types of statins and laboratory tests. Increased plasma TM levels are a marker of endothelial damage, and statins protect endothelial cells and maintain anticoagulant properties by upregulating TM gene expression and reducing 

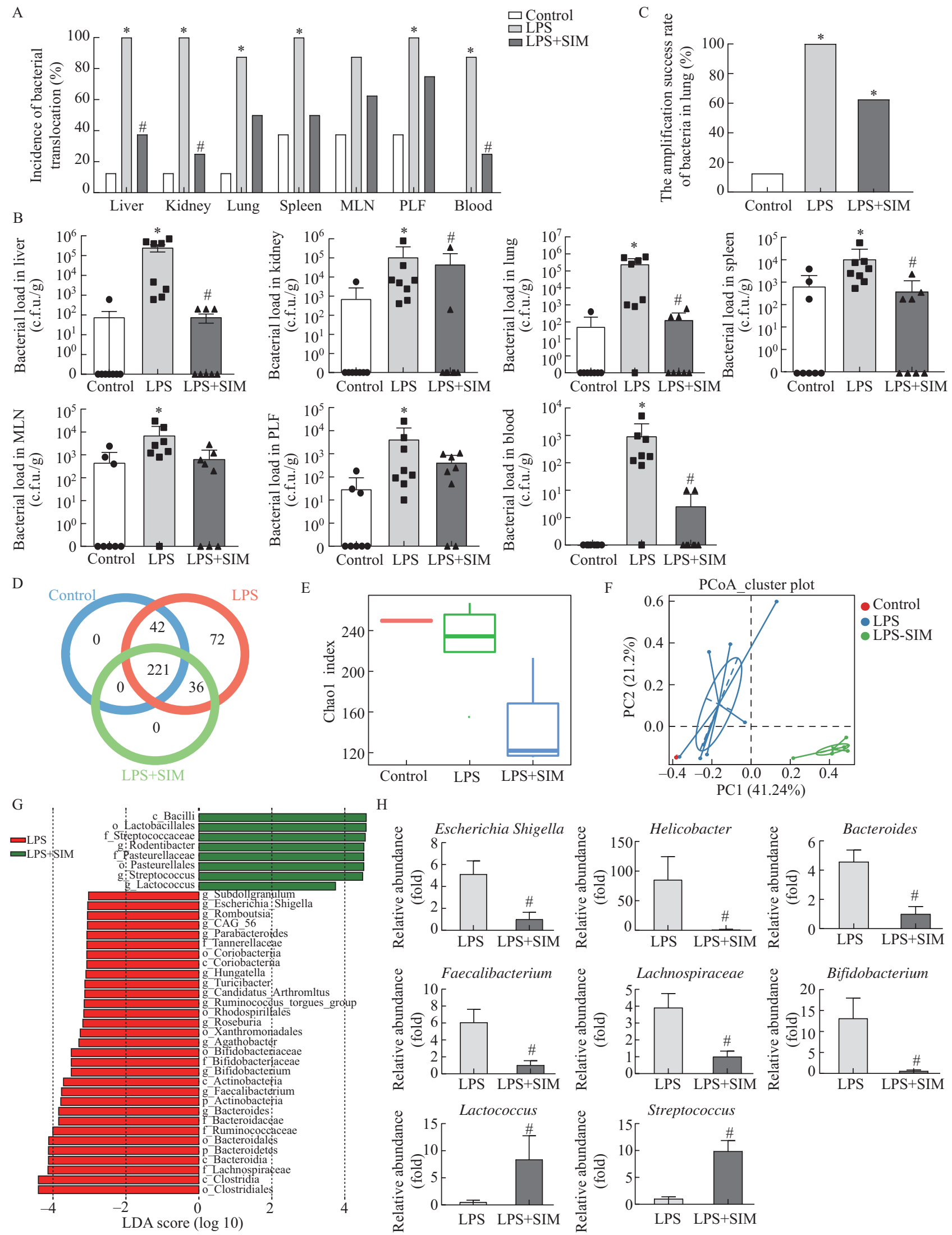

Fig. 6 Simvastatin inhibits bacterial translocation
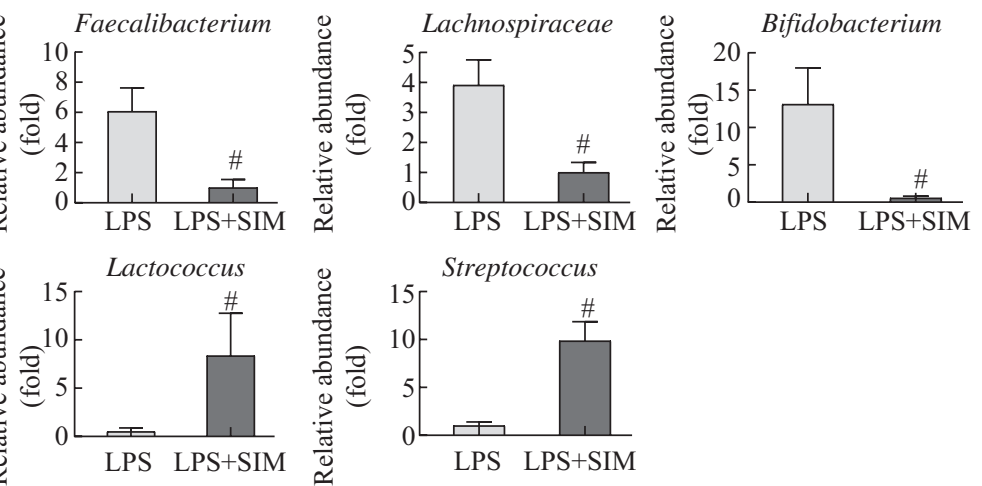

A: incidence of bacterial translocation (\%). B: Bacterial load (c.f.u./g) in the liver, kidney, lung, spleen, MLNs, PLF, and blood. C: the amplification success rate of bacteria in lungs. D: Venn diagram. E: PCoA score plot. F: Chaol index. G: distinctive gut microbiota composition revealed by LDA in the indicated groups. $\mathrm{H}$ : comparison of the relative taxonomic abundance among the indicated groups. The data are presented as the mean $\pm \operatorname{SEM}(n=8)$. ${ }^{*} P<0.05 v$ s. the control group, ${ }^{\sharp} P<0.05$ comparisons between the LPS group and the LPS+SIM group. MLNs: mesenteric lymph nodes; PLF: peritoneal lavage fluid; PCoA: principal co-ordinates analysis; LDA: linear discriminant analysis; LPS: lipopolysaccharide; SIM: simvastatin 
release $^{[23]}$. Besides, statin therapy significantly lowered the plasma PAI-1 concentration, which is consistent with the findings of a recent meta-analysis ${ }^{[24]}$. The pronounced improvement in the imbalance between coagulation and fibrinolysis in the SIM group was associated with less severe tissue damage and a lower mortality rate. A recent study also has found that preICU statin use is causally associated with a decrease in the 30-day ICU mortality and ICU length of stay compared to non-use ${ }^{[25]}$.

Long-term studies have shown that the gut plays an important role in sepsis and MODS progression ${ }^{[5,6]}$. For example, gut epithelial apoptosis is increased in sepsis, and overexpression of the antiapoptotic protein Bcl-2 can improve survival in murine sepsis models ${ }^{[18]}$. In our experiments, statins alleviated apoptosis in the intestine, accompanied by decreased caspase 3 and increased Bcl-2 expression levels.

Mucosal barrier dysfunction is the most frequent complication within the gastrointestinal tract during sepsis $^{[6]}$. TJs between intestinal epithelial cells form a barrier that selectively controls the transport of intestinal contents to the blood and prevents the entry of antigens, microorganisms, and toxins into the body. Disruption of the TJ barrier can result in increased intestinal permeability and promote bacterial stasis, bacterial overgrowth, and translocation ${ }^{[5,8]}$. The concept that LPS induces inflammatory cytokine release, which leads to increased NO production and gut injury as well as contributes to the loss of gut barrier function, is not novel ${ }^{[26,27]}$. Signaling pathways, such as the myosin light chain kinase (MLCK), protein kinase C, and Rho GTPase pathways, are also involved in the regulation of $\mathrm{TJS}^{[28-30]}$. Statins increase the expression of ZO-1 and JAM, which is possibly related to the regulation of the balance between eNOS and iNOS and related signaling pathways ${ }^{[31]}$. However, the occludin and claudin-4 levels were markedly decreased in both the LPS and SIM groups at $12 \mathrm{~h}$. We speculate that the accumulation of harmful substances in the intestine led to an increase in intestinal inflammation in the simvastatin-treated group, which in turn inhibited the expression of $\mathrm{TJ}$ proteins through the activation of MLCK $^{[6]}$. Alternatively, changes in gut microbes and specific activation of the immune system are involved in this process. For example, the probiotics Streptococcus thermophilus and Lactobacillus acidophilus can protect against TJ disruption via Rho family GTPases ${ }^{[32]}$. Notably, several TJ proteins were upregulated in simvastatin-treated Caco-2 cells, which consist of a single environment compared with the intestine.

The chemical barrier is another important player in barrier function. C3gnt is responsible for the glycosylation of intestinal mucins, thus providing an important source of growth substrates for intestinal bacteria $^{[20]}$. Muc2 can disassociate pathogenic and commensal bacteria. Muc2 $2^{--}$mice are highly susceptible to sepsis ${ }^{[5,19]}$, and lysozyme has been shown to form a high-affinity complex with bacterial LPS and inhibit its biological activity ${ }^{[33]}$. Additionally, Iap is a gut mucosal defense factor known to dephosphorylate LPS $^{[34]}$. Furthermore, the antibacterial peptides angiogenin-1, Defb-1, Reg3b, and Reg3 g have been reported to have broad-spectrum antibacterial activity and quickly kill targets to inhibit bacterial translocation and intestinal inflammation ${ }^{[35]}$.

A biological barrier composed of gut microbes is essential to maintain gut homeostasis. We found that the Bacteroidetes/Firmicutes ratio was decreased in the LPS group but restored in the SIM group, which was consistent with a recent study ${ }^{[36]}$. In the linear discriminant analysis effect size, the SIM-treated group showed an increased abundance of beneficial bacteria in the class Bacilli. This expansion was not class-wide but was due to an increase in the order of Lactobacillaceae. Studies have shown that exogenous Lactobacillus supplementation is associated with a lower incidence of sepsis in newborns and ICU patients with ventilator-associated pneumonia ${ }^{[37,38]}$. Bacteria in the genus Desulfovibrio, the major genus in the Desulfovibrionaceae family, generate hydrogen sulfide, a genotoxin and mucosal barrier-breaker, and most Desulfovibrionaceae members are reported to produce LPS and cause low-grade and chronic inflammation in obese subjects ${ }^{[39]}$. The population of these bacteria was increased in the LPS group. The further increase in Deferribacteraceae in the LPS group largely accounted for the expansion of Mucispirillum, which is known as a mucin degrader and is associated with early disruption of the colonic surface mucus layer ${ }^{[40]}$. Thus, these differences in gut microbiota might be important reasons why statins improve the prognosis of patients with sepsis or DIC.

Inflammation is another factor affecting the intestinal barrier function. However, we found that SIM did not reduce intestinal inflammation, which is contrary to the anti-inflammatory effect of statins on organs such as the lungs. This result is unique as a previous study found that statin pretreatment downregulated inflammatory genes in the liver and increased intestinal inflammation ${ }^{[41]}$. We hypothesize that SIM pretreatment reduces intestinal permeability, which suppresses the movement of intestinal toxic exudate that accumulates in the intestine and promotes upregulation of intestinal inflammatory genes. Besides, the immune system can be triggered by gut microbiota $^{[42]}$. Ligation to bacterial receptors stimulates central signaling cascades (e.g., NF-kB pathways), resulting in an immunological response and thereby regulating inflammation.

SIM reduces intestinal permeability, which leads to reduced translocation of intestinal flora. In severe 
endotoxemia, the gut is severely damaged and the levels of harmful substances and microorganisms increase, which aggravates the infections ${ }^{[8]}$. A recent study has shown that an increased intestinal permeability triggers a cascade of events that result in increased bacterial growth, bacterial translocation, and risk of sepsis ${ }^{[5]}$. On the one hand, SIM improves systemic inflammation through direct anti-inflammatory and protective endothelial effects; on the other hand, it inhibits the translocation of harmful intestinal flora and toxins to the blood and organs, thereby improving inflammation and complications like coagulopathy and organ damage.

The main limitation of our study is that the experiment failed to explore the specific mechanism by which statins increase intestinal inflammation, whether through the accumulation of harmful substances or activation of the immune system by microorganisms. Second, we failed to discover whether intestinal microbes or intestinal metabolites directly regulate the coagulation system; therefore, more in-depth mechanistic studies are required in the future. Third, while all of the animals in this study had free access to food and water throughout the experiment, it is possible that oral intake decreased after the onset of endotoxin exposure. The experiments did not examine the role of nutrition in intestinal hyperpermeability, and starvation itself can induce gut barrier dysfunction.

In conclusion, we found that in severe endotoxininduced sepsis or coagulopathy, the gut is seriously damaged. SIM pretreatment attenuates coagulopathy by enhancing anticoagulation and fibrinolysis. Although the gut participates in this process, SIM improves the intestinal barrier damage and reduces the disorder of microorganisms. With these changes, the intestinal permeability is reduced and the translocation of intestinal flora to organs and blood is inhibited. The role of SIM in the regulation of intestinal microenvironmental disorders can partly explain that ICU patients with statin use prior to admission are usually associated with a decrease in mortality.

\section{Acknowledgements}

The authors would like to thank all of the study participants and the Thrombosis and Hemostasis Laboratory of Hubei Province.

\section{Open Access}

This article is licensed under a Creative Commons Attribution 4.0 International License https://creativecommons.org/licenses/by/4.0/), which permits use, sharing, adaptation, distribution and reproduction in any medium or format, as long as you give appropriate credit to the original author(s) and the source, provide a link to the Creative Commons licence, and indicate if changes were made. The images or other third party material in this article are included in the article's Creative Commons licence, unless indicated otherwise in a credit line to the material. If material is not included in the article's Creative Commons licence and your intended use is not permitted by statutory regulation or exceeds the permitted use, you will need to obtain permission directly from the copyright holder. To view a copy of this licence, visit http://creativecommons. org/licenses/by/4.0/.

\section{Conflict of Interest Statement}

The authors state that they have no conflicts of interest.

Author Heng MEI is a member of the Editorial Board for Current Medical Science. The paper was handled by the other editor and has undergone rigorous peer review process. Author Heng MEI was not involved in the journal's review of, or decision related to, this manuscript.

\section{REFERENCES}

1 Singer M, Deutschman CS, Seymour CW, et al. The Third International Consensus Definitions for Sepsis and Septic Shock (Sepsis-3). JAMA, 2016,315(8):801810

2 Levi M, Dörffler-Melly J, Reitsma P, et al. Aggravation of endotoxin-induced disseminated intravascular coagulation and cytokine activation in heterozygous protein-C-deficient mice. Blood, 2003,101(12):48234827

3 Gando S, Shiraishi A, Yamakawa K, et al. Role of disseminated intravascular coagulation in severe sepsis. Thromb Res, 2019,178:182-188

4 Rangel-Frausto MS, Pittet D, Costigan M, et al. The natural history of the systemic inflammatory response syndrome (SIRS). A prospective study. JAMA, 1995, 273(2):117-123

5 Kumar M, Leon Coria A, Cornick S, et al. Increased intestinal permeability exacerbates sepsis through reduced hepatic SCD-1 activity and dysregulated iron recycling. Nature Commun, 2020,11(1):483

6 Fay KT, Ford ML, Coopersmith CM. The intestinal microenvironment in sepsis. Biochim Biophys Acta Mol Basis Dis, 2017,1863(10):2574-2583

7 Shimizu K, Ogura H, Goto M, et al. Altered gut flora and environment in patients with severe SIRS. J Trauma, 2016,60(1):126-133

8 MacFie J, O'Boyle C, Mitchell CJ, et al. Gut origin of sepsis: a prospective study investigating associations between bacterial translocation, gastric microflora, and septic morbidity. Gut, 1999,45(2):223-228

9 Deitch EA. Bacterial translocation or lymphatic drainage of toxic products from the gut: what is important in human beings? Surgery, 2002,131(3):241-244

10 Arnaud H, Fabien L, Vanina L, et al. Simvastatin inhibits the pro-inflammatory and pro-thrombotic effects of IL17 and TNF- $\alpha$ on endothelial cells. Ann Rheum Dis, 2013,72(5):754-760

11 Beigel Y, Fuchs J, Snir M, et al. Lovastatin therapy in hypercholesterolemia: effect on fibrinogen, hemorrheologic parameters, platelet activity, and red blood cell morphology. J Clin Pharmacol, 1991,31(6): 512-517

12 Mortensen EM, Restrepo MI, Copeland LA, et al. Impact of previous statin and angiotensin II receptor blocker use on mortality in patients hospitalized with sepsis. Pharmacotherapy, 2007,27(12):1619-1626

13 Merx MW, Liehn EA, Janssens U, et al. HMG-CoA 
reductase inhibitor simvastatin profoundly improves survival in a murine model of sepsis. Circulation, 2004, 109(21):2560-2565

14 Shih CC, Hsu LP, Liao MH, et al. Effects of SPAK on vascular reactivity and nitric oxide production in endotoxemic mice. Eur J Pharmacology, 2017,814:248254

15 He X, Zheng N, He J, et al. Gut Microbiota Modulation Attenuated the Hypolipidemic Effect of Simvastatin in High-Fat/Cholesterol-Diet Fed Mice. J Proteome Res, 2017,16(5):1900-1910

16 Cani PD, Bibiloni R, Knauf C, et al. Changes in gut microbiota control metabolic endotoxemia-induced inflammation in high-fat diet-induced obesity and diabetes in mice. Diabetes, 2008,57(6):1470-1481

17 Huse SM, Welch DM, Morrison HG, et al. Ironing out the wrinkles in the rare biosphere through improved OTU clustering. Environ Microbiol, 2010,12(7):18891898

18 Coopersmith CM, Stromberg PE, Dunne WM, et al. Inhibition of intestinal epithelial apoptosis and survival in a murine model of pneumonia-induced sepsis. JAMA, 2002,287(13):1716-1721

19 Bergstrom KS, Kissoon-Singh V, Gibson DL, et al. Muc2 protects against lethal infectious colitis by disassociating pathogenic and commensal bacteria from the colonic mucosa. PLoS Pathog, 2010,6(5):e1000902

20 Xia L. Core 3-derived O-glycans are essential for intestinal mucus barrier function. Meth Enzymol, 2010, 479:123-141

21 Franco RF, de Jonge E, Dekkers PE, et al. The in vivo kinetics of tissue factor messenger RNA expression during human endotoxemia: relationship with activation of coagulation. Blood, 2000,96(2):554-559

22 Eto M, Kozai T, Cosentino F, et al. Statin prevents tissue factor expression in human endothelial cells: role of Rho/Rho-kinase and Akt pathways. Circulation, 2002,105(15):1756-1759

23 Pathak R, Ghosh SP, Zhou D, et al. The Vitamin EAnalog Gamma-Tocotrienol (GT3) and Statins Synergistically Up-Regulate Endothelial Thrombomodulin (TM). Int J Mol Sci, 2016,17(11):1937

24 Sahebkar A, Catena C, Ray KK, et al. Impact of statin therapy on plasma levels of plasminogen activator inhibitor-1. A systematic review and meta-analysis of randomised controlled trials. Thromb Haemost, 2016, 116(1):162-171

25 Chinaeke EE, Love BL, Magagnoli J, et al. The impact of statin use prior to intensive care unit admission on critically ill patients with sepsis. Pharmacotherapy, 2021,41(2):162-171

26 Han X, Fink MP, Delude RL. Proinflammatory cytokines cause $\mathrm{NO}^{*}$-dependent and -independent changes in expression and localization of tight junction proteins in intestinal epithelial cells. Shock, 2003,19(3):229-237

27 Han X, Fink MP, Yang R, et al. Increased iNOS activity is essential for intestinal epithelial tight junction dysfunction in endotoxemic mice. Shock, 2004,21(3): 261-270
28 Farhadi A, Keshavarzian A, Ranjbaran Z, et al. The role of protein kinase $\mathrm{C}$ isoforms in modulating injury and repair of the intestinal barrier. J Pharmacol Exp Ther, 2006,316(1):1-7

29 Scott KG, Meddings JB, Kirk DR, et al. Intestinal infection with Giardia spp. reduces epithelial barrier function in a myosin light chain kinase-dependent fashion. Gastroenterology, 2002,123(4):1179-1190

30 Peng H, Luo P, Li Y, et al. Simvastatin alleviates hyperpermeability of glomerular endothelial cells in early-stage diabetic nephropathy by inhibition of RhoA/ ROCK1. PLoS ONE, 2013,8(11):e80009

31 Gong X, Ma Y, Ruan Y, et al. Long-term atorvastatin improves age-related endothelial dysfunction by ameliorating oxidative stress and normalizing eNOS/ iNOS imbalance in rat aorta. Exp Gerontol, 2014,52:917

32 Trivedi K, Barrett KE, Resta-Lenert SC. Probiotic inhibition of the entry of enteroinvasive E. coli into, human intestinal epithelial cells involves both Rho-dependent and -independent pathways. Gastroenterology, 2003,124(4):A106-A106

33 Takada K, Ohno N, Yadomae T. Detoxification of lipopolysaccharide (LPS) by egg white lysozyme. FEMS Immunol Med Microbiol, 1994,9(4):255-263

34 Estaki M, DeCoffe D, Gibson DL. Interplay between intestinal alkaline phosphatase, diet, gut microbes and immunity. World J Gastroenterol, 2014,20(42):15 65015656

35 Vaishnava S, Yamamoto M, Severson KM, et al. The antibacterial lectin RegIIIgamma promotes the spatial segregation of microbiota and host in the intestine. Science, 2011,334(6053):255-258

36 Caparrós-Martín JA, Lareu RR, Ramsay JP, et al. Statin therapy causes gut dysbiosis in mice through a PXRdependent mechanism. Microbiome, 2017,5(1):95

37 Panigrahi P, Parida S, Nanda NC, et al. A randomized synbiotic trial to prevent sepsis among infants in rural India. Nature, 2017,548(7668):407-412

38 Shimizu K, Yamada T, Ogura H, et al. Synbiotics modulate gut microbiota and reduce enteritis and ventilator-associated pneumonia in patients with sepsis: a randomized controlled trial. Crit Care, 2018,22(1):239

39 Wei ZS, Augusto LA, Zhao LP, et al. Desulfovibrio desulfuricans isolates from the gut of a single individual: structural and biological lipid A characterization. FEBS Lett, 2014,589(1):165-171

40 Belzer C, Gerber GK, Roeselers G, et al. Dynamics of the microbiota in response to host infection. PLoS ONE, 2014,9(7):e95534

41 Nolan JA, Skuse P, Govindarajan K, et al. The influence of rosuvastatin on the gastrointestinal microbiota and host gene expression profiles. Am J Physiol Gastrointest Liver Physiol, 2017,312(5):G488-G497

42 Cekanaviciute E, Yoo BB, Runia TF, et al. Gut bacteria from multiple sclerosis patients modulate human $\mathrm{T}$ cells and exacerbate symptoms in mouse models. Proc Natl Acad Sci USA, 2017,114(40):10713-10718

(Received Apr. 9, 2021; accepted Jul. 4, 2021) 